
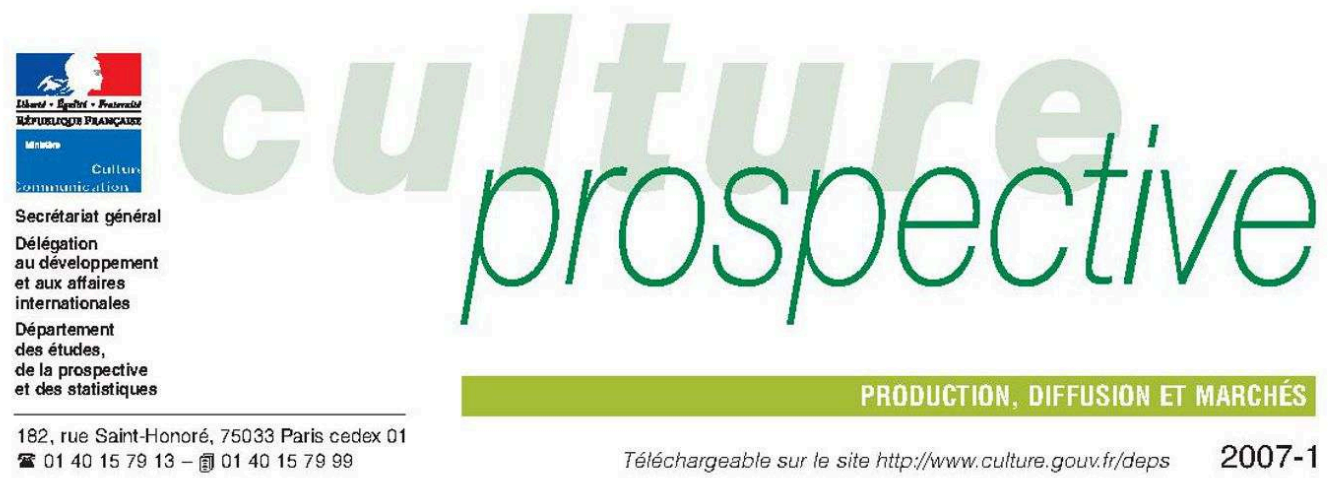

\title{
Musique enregistrée et numérique : quels scénarios d'évolution de la filière?
}

Marc Bourreau, Michel Gensollen et François Moreau*

\section{Avant-propos}

Le DEPS inaugure une nouvelle collection, «Culture prospective ». Elle est consacrée à l'investigation d'hypothèses, à des mises en relation originale et à des travaux de prospective sur des champs connus comme sur des terrains plus marginaux ou expérimentaux. Traduction d'études et de travaux menés par le DEPS, elle peut tout autant accueillir des recherches en cours.

À partir d'un travail d'enquête auprès d'acteurs de l'ensemble des segments de la filiere française de la musique enregistrée, les auteurs ont entrepris de dégager sur un fond théorique de l'économie numérique plusieurs scénarios d'évolution de l'industrie des phonogrammes. Issus du croisement des effets économiques et d'usages de la dématérialisation des biens numériques et des logiques d'information sur ces biens, ces scénarios dessinent un cadre cohérent de l'évolution de la structure du marché et de la filière de la musique enregistrée, en réponse à la mutation qu'ils traversent.

P. C.
La filière de la musique enregistrée - qu'on peut définir comme l'ensemble du système social, réglementaire, technique et économique qui met en rapport une offre musicale originale avec des consommateurs qui sont disposés à l'écouter - traverse des mutations importantes, généralement décrites sous le terme de «révolution numérique ${ }^{1} \gg$. Le caractère reproductible des œuvres qu'elle produit, diffuse et commercialise a été amplifié depuis plus de deux décennies par une numérisation croissante des contenus musicaux couplée à un développement très important des possibilités de diffusion numérique. Ces changements n'ont pas seulement influé substantiellement sur la reproductibilité des contenus; ils ont aussi produit des effets à chaque stade de la filière, de la phase créative en amont à la phase de mise à disposition au consommateur final en aval.

En réponse à ces mutations, plusieurs scénarios théoriques d'évolution à moyen terme de la filière peuvent être esquissés à partir, notamment, d'entretiens menés avec des professionnels de la filière représentatifs de plusieurs métiers : labels, éditeurs musicaux, studios d'enregistrement, distributeurs et producteurs de spectacles.

Un bref rappel des caractéristiques économiques de la numérisation des contenus permet de simplifier l'exposi-

\footnotetext{
* Respectivement maître de conférences (ENST, département sciences Gconomiques et sociales, et CREST-Lel), chercheur associé au département sciences économiques et sociales de Télecom Paris et maître de conférences au laboratoire d'econométrie du Conservatoire national des arts et métiers. Parmi les recherches les plus récentes de ces trois auteurs, certaines portent sur l'économie numérique.

arts et metiers. Pami les recherches les plus recentes de ces trois auteurs, certaines portent sur l'economie numerique.
1. Marc Bourreavet Michel Gensollen, «L'impact d'internet et des technologies de l'information et de la communication sur l'industrie de

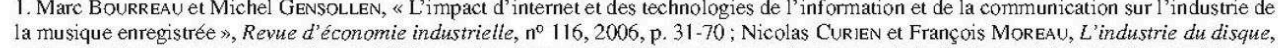
Paris, La Découverte, coll. "Repères », 2006; Martin Pejtz et Patrick WAELbroeck, "An Economist's Guide to Digital Music", CESifo Economic Studies, 2005, $51(2-3)$, p. 359-428; Philippe CHANTEFIE et Alain Le DiBERDER, Révolution numérique et industries culturelles, Paris, La Découverte, coll. «Repères», 2005 ; André NiCOLAs et Vicior CONRADSSON, Les marchés numériques de la musique, Paris, Observatoire des usages numériques culturels, Ministère de la culture et de la communication, 2005.
} 


\section{Musique enregistrée et numérique : quels scénarios d'évolution de la filière?}

\section{Marc Bourreau, Michel Gensollen et François Moreau}

Éditeur : Département des études, de la prospective et des statistiques

Lieu d'édition : Paris

Année d'édition : 2007

Date de mise en ligne : 21 septembre 2015

Collection : Culture prospective

ISBN électronique : 9782111398009

\section{QboOoks}

http://books.openedition.org

\section{Édition imprimée}

Date de publication : 1 avril 2007

Nombre de pages : 16

\section{Référence électronique}

BOURREAU, Marc ; GENSOLLEN, Michel ; et MOREAU, François. Musique enregistrée et numérique quels scénarios d'évolution de la filière ? Nouvelle édition [en ligne]. Paris : Département des études, de la prospective et des statistiques, 2007 (généré le 25 avril 2021). Disponible sur Internet : <http:// books.openedition.org/deps/852>. ISBN : 9782111398009 

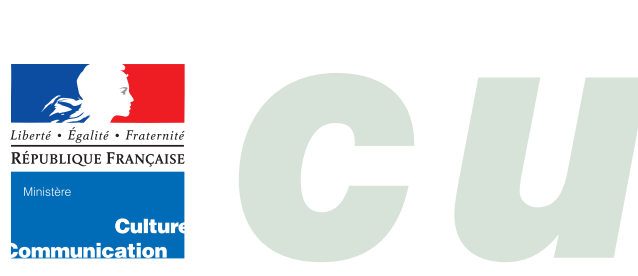

Secrétariat général

Délégation

au développement

et aux affaires

internationales

Département

des études,

de la prospective

et des statistiques

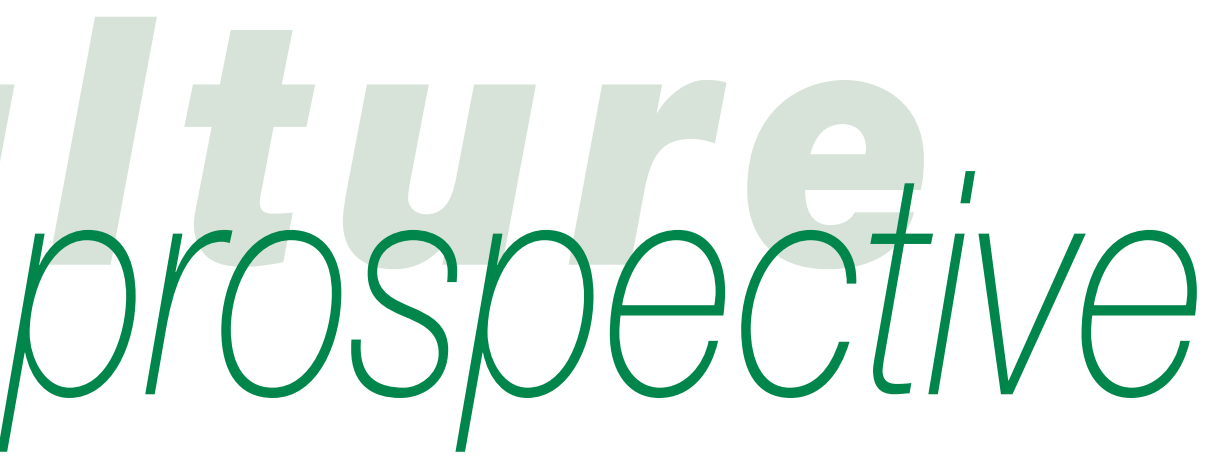

\title{
Musique enregistrée et numérique : quels scénarios d'évolution de la filière?
}

\author{
Marc Bourreau, Michel Gensollen et François Moreau*
}

\section{Avant-propos}

Le DEPS inaugure une nouvelle collection, «Culture prospective ». Elle est consacrée à l'investigation d'hypothèses, à des mises en relation originale et à des travaux de prospective sur des champs connus comme sur des terrains plus marginaux ou expérimentaux. Traduction d'études et de travaux menés par le DEPS, elle peut tout autant accueillir des recherches en cours.

À partir d'un travail d'enquête auprès d'acteurs de l'ensemble des segments de la filière française de la musique enregistrée, les auteurs ont entrepris de dégager sur un fond théorique de l'économie numérique plusieurs scénarios d'évolution de l'industrie des phonogrammes. Issus du croisement des effets économiques et d'usages de la dématérialisation des biens numériques et des logiques d'information sur ces biens, ces scénarios dessinent un cadre cohérent de l'évolution de la structure du marché et de la filière de la musique enregistrée, en réponse à la mutation qu'ils traversent.

P. C.
La filière de la musique enregistrée - qu'on peut définir comme l'ensemble du système social, réglementaire, technique et économique qui met en rapport une offre musicale originale avec des consommateurs qui sont disposés à l'écouter - traverse des mutations importantes, généralement décrites sous le terme de «révolution numérique $^{1} »$. Le caractère reproductible des œuvres qu'elle produit, diffuse et commercialise a été amplifié depuis plus de deux décennies par une numérisation croissante des contenus musicaux couplée à un développement très important des possibilités de diffusion numérique. Ces changements n'ont pas seulement influé substantiellement sur la reproductibilité des contenus ; ils ont aussi produit des effets à chaque stade de la filière, de la phase créative en amont à la phase de mise à disposition au consommateur final en aval.

En réponse à ces mutations, plusieurs scénarios théoriques d'évolution à moyen terme de la filière peuvent être esquissés à partir, notamment, d'entretiens menés avec des professionnels de la filière représentatifs de plusieurs métiers : labels, éditeurs musicaux, studios d'enregistrement, distributeurs et producteurs de spectacles.

Un bref rappel des caractéristiques économiques de la numérisation des contenus permet de simplifier l'exposi-

\footnotetext{
* Respectivement maître de conférences (ENST, département sciences économiques et sociales, et CREST-LEI), chercheur associé au département sciences économiques et sociales de Télécom Paris et maître de conférences au laboratoire d'économétrie du Conservatoire national des arts et métiers. Parmi les recherches les plus récentes de ces trois auteurs, certaines portent sur l'économie numérique.

1. Marc Bourreau et Michel Gensollen, «L'impact d'internet et des technologies de l'information et de la communication sur l'industrie de la musique enregistrée », Revue d'économie industrielle, $\mathrm{n}^{\circ}$ 116, 2006, p. 31-70 ; Nicolas CURIEN et François MoREAU, L'industrie du disque, Paris, La Découverte, coll. «Repères », 2006 ; Martin Peitz et Patrick WAElbroeck, "An Economist's Guide to Digital Music", CESifo Economic Studies, 2005, 51(2-3), p. 359-428; Philippe ChANTEPIE et Alain Le Diberder, Révolution numérique et industries culturelles, Paris, La Découverte, coll. «Repères », 2005 ; André Nicolas et Victor CONRADSsON, Les marchés numériques de la musique, Paris, Observatoire des usages numériques culturels, Ministère de la culture et de la communication, 2005.
} 
tion de cinq scénarios contrastés qui peuvent être rapprochés d'un certain nombre de modèles d'affaires émergents.

\section{RAPPEL SUR L'ÉCONOMIE DE LA NUMÉRISATION}

Trois variables structurent les scénarios proposés et leurs conséquences en termes de bien-être $\operatorname{social}^{2}$ : la nature technico-économique des fichiers numérisés qui transforme la mise en valeur de la musique; le mode de confrontation entre l'offre et la demande de musique car, la musique étant un bien d'expérience ${ }^{3}$, les consommateurs éventuels doivent être informés et la consommation initiée par un système complexe en cours de transformation ; la façon, enfin, dont est considéré le produit «musique enregistrée » : s'agit-il d'un produit comme un autre ou bien la musique, et plus généralement les produits culturels, remplissent-ils un rôle social spécifique qui justifierait un encadrement adapté ?

\section{Type de valorisation des fichiers numériques}

\section{Les caractéristiques d'un bien numérique}

\section{Non-rivalité et permanence}

Un bien numérisé se réduit à un fichier informatique (une suite d'octets) à un moment quelconque entre la production et la consommation. Généralement, la production initiale et la consommation finale ne sont pas numérisées. Ainsi, dans le cas de la musique, les artistes jouent d'instruments (qui peuvent d'ailleurs être numériques) et les consommateurs écoutent une musique recréée par les appareils nécessaires pour transformer le flux numérique en sons.

Dans la mesure où les fichiers informatiques sont reproductibles à des coûts marginaux pratiquement nuls et des coûts moyens très faibles, les équipements de copie étant intégrés de façon standard dans la plupart des micro-ordinateurs, la musique

\footnotetext{
2. Traditionnellement en économie, le bien-être social est mesuré par la somme du «surplus des producteurs » et du «surplus des consommateurs ». Le surplus des producteurs est la différence entre ce que ces producteurs touchent pour les biens et services qu'ils produisent et le coût de les offrir. Le surplus des consommateurs est la différence entre ce que les consommateurs seraient disposés à payer pour les biens et services existants et ce qu'ils ont en réalité à payer pour en bénéficier.

3. Un bien d'expérience est un produit ou un service dont les caractéristiques telles que la qualité et le prix sont difficiles à observer par avance, c'est-à-dire avant consommation.
}

numérisée est devenue un bien techniquement non rival : un fichier peut circuler d'utilisateur en utilisateur sans coûts substantiels. La diffusion du réseau internet, en particulier du réseau à haut débit, et la réduction des prix des équipements informatiques permettent ainsi un accès universel à la musique comme aux images animées.

Dans le cas des biens numériques, il convient de distinguer non-rivalité et permanence, caractéristiques différentes mais parfois confondues dans la mesure où la gestion numérique des droits sur les œuvres musicales cherche souvent à traiter les deux questions à la fois ${ }^{4}$. Un bien est permanent si son utilisation ne le détruit pas ni, même, n'en modifie les caractéristiques techniques. Les biens permanents s'apparentent donc aux biens d'équipement qui, eux non plus, ne sont pas détruits dans la période considérée 5 .

S'il est peu douteux que non-rivalité et permanence représentent un gain de bien-être social, encore faut-il organiser cette nouvelle abondance, dont la première conséquence est de s'opposer au fonctionnement traditionnel des marchés qui s'étaient définis autour de supports physiques (pour les contenus) et de médias de masse (pour la métainformation, c'est-à-dire l'information des consommateurs sur les œuvres disponibles et leur qualité).

Afin de maintenir la valeur des contenus, une première réaction a pu consister à verrouiller techniquement les fichiers par des mesures techniques de protection afin de limiter leur copie et/ou leur usage, dans le temps ou en nombre d'écoutes. On demande ainsi à la technique de réduire les avantages qu'elle a spontanément apportés afin de maintenir inchangés les modèles d'affaires.

\section{Une non-rivalité technique ou économique?}

Un point important est la distinction qui doit être faite entre non-rivalité « technique » et non-rivalité « effective », qui s'étend d'ailleurs à la permanence. Dans certains cas, un bien peut se copier et se diffuser sans coûts, ou bien ne pas disparaître lors de la consommation et conserver néanmoins une

\footnotetext{
4. La gestion numérique des droits (Digital Rights Management en anglais ou $D R M$ ) est un terme parapluie qui fait référence à toute technologie utilisée par les éditeurs ou les ayants droit (d'auteur) pour contrôler l'accès à et l'usage de données numériques ou de matériel informatique, ainsi qu'aux restrictions associées aux cas particuliers de travaux ou de dispositifs numériques. Pour faciliter la lecture, le terme DRM sera plusieurs fois repris par la suite.

5. On notera, toutefois, que les biens d'équipement s'usent et sont onéreux à entretenir, alors que les biens numériques ne s'usent ni ne s'entretiennent. Il est vrai qu' un progrès technique rapide induit une obsolescence analogue à une sorte d'usure : les supports des fichiers et les lecteurs correspondants se démodent rapidement si bien qu'une «discothèque numérique » doit être recopiée de temps en temps afin de suivre le progrès des équipements informatiques.
} 
valeur marchande. Un exemple classique est celui d'une information qui pointe vers un bien rival ; cette information a clairement la valeur de ce bien rival $^{6}$. D'une façon générale, il convient de distinguer non-rivalité technique et externalités de consommation : une information, même si elle est non rivale, peut être telle que la consommation des uns porte conséquence sur l'utilité de la consommation par d'autres. Dans ce cas, le consommateur qui possède une information à externalité négative ${ }^{7}$ ne sera pas incité à la diffuser.

Dans le domaine des biens culturels, les externalités de consommation sont généralement positives : un consommateur ne voit pas son utilité diminuer, bien au contraire, lorsqu'il rend disponible une œuvre et en étend la consommation; tout d'abord, parce qu' on peut aimer faire connaître ce qu'on apprécie mais surtout parce qu'en contribuant au succès d'une œuvre, on peut rendre plus probables la composition et la diffusion d'œuvres semblables. Toutefois, dans les cas, assez rares, où la consommation de biens culturels se réduit à une consommation ostentatoire, les consommateurs ne sont pas incités à copier et à diffuser des fichiers puisque ceux-ci jouent le rôle de biens-signaux dont la possession est source de prestige.

Même si la diffusion ne nuit pas à celui qui partage un bien non rival, encore faut-il qu'un tel bien présente une certaine utilité pour les autres consommateurs. Il peut se faire en effet qu'un bien soit « adressé », en ce sens qu'il a été précisément adapté à un seul utilisateur. Dans le cas des biens ordinaires, une paire de lunettes fournit l'exemple d'un bien adressé, bien pour lequel il n'y a ni vol à craindre ni marché d'occasion à organiser.

Dans le cas des biens non rivaux, les œuvres qui ne s'adressent qu'à un consommateur particulier ou, de façon plus réaliste, à des audiences très étroites,

6. Éric Raymond en a donné une formulation précise dans The Magic Cauldron, 1999, http://www.catb.org/ esr/writings/cathedralbazaar/magic-cauldron/, texte sur le free software qui insiste sur le sens du mot free (ouvert mais pas nécessairement gratuit) : "There is another myth, equal and opposite to the factory-model delusion, which often confuses peoples' thinking about the economics of open-source software. It is that 'information wants to be free'. This usually unpacks to a claim that the zero marginal cost of reproducing digital information implies that its clearing price ought to be zero. The most general form of this myth is readily exploded by considering the value of information that constitutes a claim on a rivalrous good - a treasure map, say, or a Swiss bank account number, or a claim on services such as a computer account password. Even though the claiming information can be duplicated at zero cost, the item being claimed cannot be. Hence, the non-zero marginal cost for the item can be inherited by the claiming information."

7. On entend ici par «bien à externalité négative » (respectivement positive) le fait que, pour un consommateur donné, l'utilité du bien diminue (respectivement augmente) lorsque d'autres utilisateurs consomment eux aussi ce bien. C'est par exemple le cas d'un bien avec encombrement, comme un réseau routier à l'heure de pointe. sont peu utiles à copier et à diffuser au-delà de cette audience. Leurs marchés sont moins exposés au contournement et la non-rivalité de telles œuvres peut être considérée comme sans conséquences. La permanence technique peut être, elle aussi, contrecarrée par des externalités négatives ou un adressage précis; il s'agit cette fois-ci d'externalités ou d'adressage temporels.

En effet, certains biens perdent une part de leur utilité après leur consommation tandis que d'autres la conservent : un film, généralement, n'est regardé qu'une fois, même si parfois certains consommateurs peuvent prendre plaisir à le revoir. Dans le cas de la musique, inversement, l'utilité de l'écoute d'une même pièce peut se maintenir longtemps et même aller en croissant. Des stratégies d'adressage temporel peuvent venir diminuer l'intérêt de cette permanence de base; c'est le cas lorsqu'un bien n'est utile que pendant très peu de temps ou avant une date déterminée (phénomène de mode, par exemple).

Dans le tableau 1, l'ensemble des biens numériques envisageables est représenté en distinguant pour les deux critères considérés (non-rivalité et permanence) les situations où le service fourni par le progrès technique est contrecarré, soit par des moyens juridiques et techniques tels que des mesures de protection sous DRM - pour faire court, des DRM - soit par des stratégies portant sur la nature économique du bien.

\section{Tableau 1 - Diversité des biens numériques}

\begin{tabular}{|c|c|c|}
\hline & \multicolumn{2}{|c|}{ Protection } \\
\hline & $\begin{array}{l}\text { juridique } \\
\text { et technique }\end{array}$ & économique \\
\hline \multicolumn{3}{|l|}{ Non-rivalité } \\
\hline copie sans coûts & $\begin{array}{l}\text { DrM de copie } \\
\text { perte d'inter- } \\
\text { opérabilité }\end{array}$ & $\begin{array}{l}\text { Adressage } \\
\text { personnel : niche } \\
\text { Externalités: } \\
\text { ostentation }\end{array}$ \\
\hline \multicolumn{3}{|l|}{ Permanence } \\
\hline non-usure & $\begin{array}{l}\text { DRM } \\
\text { de péremption }\end{array}$ & $\begin{array}{l}\text { Adressage } \\
\text { temporel : mode }\end{array}$ \\
\hline
\end{tabular}

\section{Les stratégies génériques d'extraction de valeur}

Les principales stratégies génériques que les entreprises peuvent mettre en œuvre pour valoriser les contenus dans un environnement numérique sont de trois types : la protection directe des contenus numériques par des DRM de copie et/ou de péremption, la protection indirecte des contenus par divers procédés portant sur la nature même des œuvres diffusées et le déplacement de la valeur des contenus vers des consommations liées. 
Deux types de procédés permettent la protection indirecte des contenus :

- le renouvellement fréquent des contenus. Les effets de mode peuvent être un moyen efficace pour rendre éphémères les biens culturels, en particulier dans un modèle de promotion par les médias de masse. On notera toutefois que les fichiers musicaux, mais aussi les fichiers vidéo de film ou de série télévisée, peuvent être téléchargés sur les réseaux d'échange de pair à pair très peu de temps après leur sortie, ce qui limite la portée directe d'une telle stratégie de renouvellement. Toutefois, les échanges sur ces réseaux pourraient devenir à terme nécessaires au déclenchement des phénomènes de mode ;

- la vente par ensembles cohérents. Il s'agit de définir, soit un produit complexe constitué d'un paquet de biens (bundle), soit une source homogène de biens successifs. Dans ce dernier cas, l'utilité de chaque bien séparément est assez faible et le consommateur doit suivre l'ensemble des productions pour pleinement recueillir l'utilité offerte par la source. C'est, dans le domaine des images animées, le cas des séries qui, aux ÉtatsUnis, peuvent s'étendre sur plusieurs dizaines d'heures pour chaque saison, une même série pouvant, en cas de succès, se renouveler de saison en saison (on gagne ainsi deux ordres de grandeur en passant du produit « film de deux heures » à une série télévisée de deux cents heures). Dans ce cas, le piratage de certains épisodes, loin de nuire à la mise en valeur de la source, aide à la diffusion des épisodes, d'où la facilité avec laquelle ces fichiers se trouvent sur les réseaux de pair à pair. Dans le cas de la musique, une telle stratégie consiste à offrir des produits globaux, soit par abonnement, soit par collection (voir par exemple des offres comme «Tout Mozart en $170 \mathrm{CD} »)$. Certains labels musicaux dits «indépendants » cherchent ainsi à constituer une production cohérente, formée cependant d'artistes et de genres très différents. Ces labels espèrent que les clients prendront à terme leurs décisions d'achat plutôt à partir d'une appréciation globale de la production du label qu'à partir du nom de tel ou tel artiste. On notera, pour mémoire, deux types de stratégies déjà très utilisées dans le cas de la musique : d'une part, les stratégies de niche consistant à adapter très finement les produits pour des audiences très étroites (sur internet, des niches locales peuvent s'organiser rapidement en communautés étendues), d'autre part, les stratégies de développement de produits ostentatoires.
Deux types de procédés permettent le déplacement de la valeur des contenus vers des consommations liées :

- la stratégie de déplacement de la valeur vers des biens rivaux. Une telle stratégie vise à lier un contenu libre à des biens rivaux utiles, voire nécessaires, pour une consommation pleinement satisfaisante. Dans le cas de la musique, les textes de présentation, les livrets, les partitions, les clips, etc. peuvent jouer ce rôle et donner un support physique aux achats de contenus ouverts. Les marchés de l'accès à internet haut débit, des appareils de lecture (baladeurs numériques, téléphones portables, etc.) constituent également des biens liés rivaux ;

- la stratégie de déplacement de la valeur vers la méta-information. Il s'agit dans ce cas de jouer sur le fait que les biens culturels sont des biens d'expérience et que, même si les contenus sont désormais techniquement non rivaux, les métainformations liées sont adressées à un consommateur particulier, par exemple, dans le cas des recommandations personnalisées d'un site de vente. Néanmoins, le caractère même de la métainformation se prête mal au recueil de la disposition à payer des consommateurs : la fourniture demande en effets des échanges confiants et une connaissance intime qui cadrent mal avec des considérations sordides. Dans certains scénarios, on peut envisager que la méta-information ne se limite pas, pour un consommateur, aux informations nécessaires pour découvrir un bien culturel qui lui plaise mais s'étende aux informations nécessaires pour l'apprécier pleinement ou, éventuellement, l'adapter à ses goûts particuliers (formation d'un capital culturel).

Dans la constitution des scénarios, nous retiendrons, pour les stratégies de mise en valeur des contenus, deux stratégies principales : les stratégies de protection, que nous particulariserons selon les scénarios en «protection technique » ou «protection économique », et les stratégies de déplacement, particularisées selon les scénarios en «déplacement vers des biens rivaux » ou « déplacement vers la méta-information ».

On notera les stratégies de protection par «P.v » pour rappeler qu'il s'agit de stratégies de prix élevés et de faibles quantités vendues et les stratégies de déplacement par «p.Z» pour rappeler qu'il s'agit de la vente de produits dérivés ou même de la fourniture gratuite de ces produits avec un recueil indirect de la valeur, par exemple par la publicité dans le cas de marchés dits «bifaces » ou à deux versants. 


\section{Le rôle \\ de la méta-information}

Les biens culturels, et singulièrement les biens musicaux, ont ceci de particulier que l'offre et la demande ne se confrontent pas sur un marché classique mais dépendent d'un système de guidage complexe qui doit préparer leur adaptation qualitative. En quelque sorte, l'offre et la demande ne réagissent l'une avec l'autre qu'en présence de catalyseurs, qu'avec l'aide d'une diversité d'agents qui informent, préparent, choisissent, acculturent, sélectionnent, etc.

L'équilibre du marché dépend moins des biens disponibles et des utilités des consommateurs que du système technico-économique, désigné par le terme "prescription», qui permet la rencontre effective d'une offre qui s'adapte et d'une demande qui se forme et évolue.

Le système de prescription peut être caractérisé par le croisement de deux variables :

- la sélection des œuvres : celle-ci prend place avant la phase de production; il s'agit pour des professionnels de trier des ouvres achevées afin de décider celles qui devront être produites, ou d'orienter des artistes pendant la phase de composition ; nous supposerons dans la suite soit qu'une telle phase de sélection, effectuée par des professionnels et des experts, joue un rôle important, soit, au contraire, qu'il n'y a pas de système professionnel de sélection des œuvres ou que cette sélection joue un rôle secondaire ;

- la promotion des œuvres : celle-ci prend place après la phase de production; il s'agit de faire connaitre les œuvres et, en quelque sorte, de produire la demande correspondant à l'offre; nous supposerons que la promotion peut être, soit centralisée (promotion par les médias de masse comme la radio et la télévision), soit décentralisée (promotion par le bouche-à-oreille, direct ou sur internet).

Quatre structures méta-informationnelles émergent de cette caractérisation: modèles Star system, Push, Pull structuré et Pull libre (voir tableau 2 et, de manière plus détaillée, encadré ci-contre).

\section{Tableau 2 - Les quatre structures méta-informationnelles}

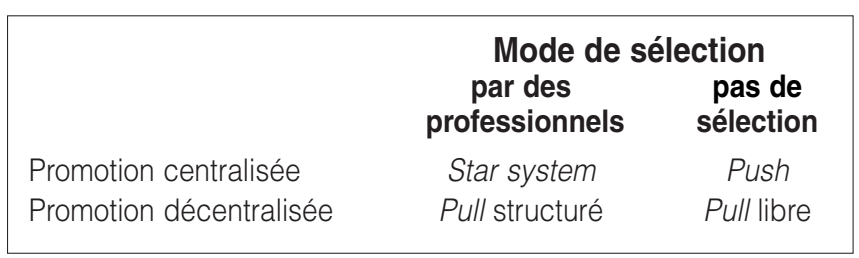

Les quatre structures méta-informationnelles de la filière de la musique enregistrée

- Le modèle Star system conjugue une promotion centralisée par les médias de masse avec une sélection professionnelle, par des labels indépendants qui peuvent assurer à la fois diversité et qualité. C'est à peu près la situation actuelle même si l'on peut percevoir une tendance à une évolution vers le modèle Push.

- Le modèle Push est associé à l'absence de sélection joint à la promotion centralisée que certains associent à un modèle «Star $A c$ » caractérisé par une baisse de qualité générale, une uniformité de produits, un formatage des goûts, etc. Le modèle Push est adapté aux biens de mode ou d'ostentation dont l'utilité ne dépend pas de leur qualité intrinsèque et pour lesquels aucune sélection n'est vraiment nécessaire. Les médias de masse se montrent efficaces pour fabriquer et imposer des vedettes éphémères et pour promouvoir des œuvres standardisées par des diffusions répétées.

- Le modèle Pull structuré, proche du modèle actuel des labels indépendants, se caractérise par une promotion décentralisée, par la fourniture de conseils personnalisés, par exemple sur les sites de vente de produits culturels (comme Amazon), par un bouche-à-oreille en ligne, c'est-à-dire par un système critique réparti (par exemple, à partir de blogs) permettant l'émergence de biens culturels adaptés à de petites collectivités. Certains standards de qualité sont néanmoins encore assurés par une sélection professionnelle, en particulier les standards techniques. $\mathrm{Ce}$ modèle peut permettre, notamment par le biais d'internet, un traitement de la méta-information plus diversifié que celui des médias de masse et le respect des schémas actuels de mise en valeur des contenus par des supports rivaux.

- Le modèle Pull libre repose sur la possibilité, pour des artistes, d'entrer directement en contact avec leur public, sans sélection professionnelle, sans promotion centralisée. Pour certains, il s'agit là d'une utopie et des médiations sans doute peu différentes des formes actuelles se remettront rapidement en place; ainsi, sur internet (blogs, sites de type MySpace), les visites des internautes se font vers les artistes très connus, qui sont justement ceux qui sont promus par les médias de masse. Pour d'autres, même si internet est un média et donc opère une sélection dans ce qu'il transmet, cette sélection est différente de celle qu'effectuent les médias de masse : statiquement, elle permettrait plus de diversité (ce qu'on désigne, dans ce contexte, par l'expression de «longue traîne ", long tail* ${ }^{\star}$ et, dynamiquement, une recomposition de la production selon les réactions de l'offre.

* L'expression long tail vient d'un article de Chris Anderson dans la revue Wired (octobre 2004, http://www.wired.com/wired/archive/12.10/tail.html). Prenant l'exemple des avis et des critiques sur Amazon, l'auteur faisait remarquer que les sites commerciaux sur internet, parce qu'ils offrent une diversité bien plus grande que les boutiques physiques, font l'essentiel de leur chiffre d'affaires sur des articles peu vendus et non, comme il était classique, sur les best-sellers (la décroissance des ventes avec le rang est en loi puissance plutôt qu'exponentielle).

\section{L'utilité individuelle et sociale de la musique}

Dans une vision prospective, trois perspectives peuvent être raisonnablement adoptées pour définir une œuvre musicale. Elle peut être considérée comme un bien de consommation, comme la plupart des biens qu'on trouve sur le marché, à la différence près qu'il s'agit d'un bien d'expérience; comme un bien social, pour lequel la formation de la demande dépend plus d'interactions sociales et de phénomènes de mode que de la nature du bien 
lui-même; comme un bien culturel, qui a ceci de particulier que les producteurs s'estiment les mieux placés pour déterminer les paramètres de sa production et de sa consommation. Dans cette perspective, les activités culturelles sont associées à des activités tutélaires comme dans les domaines de l'enseignement et de la santé.

La représentation que l'on se fait des œuvres musicales, biens d'expérience, sociaux ou culturels, conditionne le jugement porté sur telle ou telle évolution technique, juridique ou économique.

Selon le métier qu'ils exercent, les acteurs de la filière de la musique enregistrée participent plus ou moins de l'une ou l'autre vision, les labels et studios se plaçant plutôt dans une optique de biens culturels, les autres métiers se répartissant entre les deux autres selon la confiance qu'ils font au pouvoir de prescription des médias. Ainsi, la variable « représentation de l'utilité de la musique » permetelle de juger chaque scénario, selon l'acteur. Elle pourrait permettre également d'indiquer au décideur dans quelle mesure une décision particulière s'inscrit dans une vision économique ou culturelle de la production musicale.

\section{L'œuvre musicale comme bien d'expérience}

Se représenter la musique comme un bien économique habituel suppose que chaque individu a des goûts bien définis et exogènes, soit innés, soit plutôt formés dans l'enfance mais, en tout cas, peu modifiés à moyen terme par la consommation. Les marchés devant être équipés dans la mesure où la qualité d'une œuvre n'est pas connue avant la consommation, il faut un système critique pour éclairer les consommateurs, selon qu'on imagine que la qualité d'une œuvre est «verticale», c'està-dire que tous les consommateurs partagent à peu près le même jugement, ou «horizontale ». Dans le cas - plus réaliste - où la qualité est verticale, on peut définir la localisation des œuvres et des consommateurs dans un espace de goûts, l'utilité de la consommation d'une œuvre par un certain consommateur étant d'autant plus grande que cette œuvre est proche de ses goûts.

Dans le cas de la qualité horizontale, le système prescriptif est plus performant pour informer ex post les consommateurs de l'emplacement des œuvres que pour indiquer ex ante aux artistes l'emplacement des consommateurs. Une telle modélisation est d'ailleurs confortée par les opinions de labels qui se voient naturellement dans le rôle de diffuseurs d'œuvres nouvelles vers des audiences ciblées plutôt que dans celui d'informateurs des artistes sur ce qui serait attendu par les consommateurs.
Ceux qui pensent que les œuvres musicales sont essentiellement des biens d'expérience insistent donc sur l'importance d'internet dans le développement d'un système critique efficace. En quelque sorte, ils espèrent que les contenus se numériseront lentement, pourront être protégés et, dans le même temps, qu'internet servira principalement à la diffusion et à l'échange de méta-informations.

\section{L'œuvre musicale comme bien social}

Dans le cas d'une œuvre musicale comme bien social, les biens ne sont pas consommés pour euxmêmes mais parce que les consommateurs s'imitent les uns les autres (mode) ou se servent de leur consommation pour s'envoyer des signaux (ostentation, par exemple). Le contenu même des œuvres est ainsi sans conséquence sur le bien-être social. Un système de prescription centralisé devient même plus performant qu'un système complexe de critique, qui présuppose que les œuvres sont jugées pour elles-mêmes.

En réalité, si l'œuvre musicale est un bien social, l'utilité d'une œuvre pour un consommateur dépend du fait que d'autres l'auront consommée et qu'il sera possible pour les uns et les autres de parler ensemble de leurs expériences. On notera cependant que ce type d'externalité présuppose que les consommateurs reconnaissent une qualité intrinsèque aux œuvres, ou tout au moins qu'ils s'en persuadent. On retrouve là le fait général qu'une mode, même lorsqu'elle se réduit à un phénomène d'imitation, ne peut se donner pour tel : l'absurdité d'un pur phénomène d'imitation doit au moins se voiler ${ }^{8}$ de divers prétextes.

\section{L'œuvre musicale comme bien culturel}

Une œuvre musicale comme bien culturel repose sur le fait que les goûts des consommateurs ne sont pas fixés une fois pour toutes mais varient avec l'historique de leur consommation. Certaines œuvres ont la faculté de créer leur demande ou, si l'on veut, d'attirer à eux les consommateurs dans l'espace des goûts. Il s'agit là de phénomènes complexes et les modèles dits «d'addiction » qui ont été d'abord proposés rendent mal compte de la création culturelle. Il est clair que la demande de musique n'a pas la même dynamique que la demande d'une drogue, ce qui était le schéma initial de l'article pionnier de Stigler et Becker ${ }^{9}$.

8. Sauf dans le cas de la mode vestimentaire, où le caprice est au contraire mis en scène.

9. George J. Stigler et Gary S. Becker, "De Gustibus Non Est Disputandum”, American Economic Review, 1977, 67(2), p. 76-90. 
Selon les acteurs, selon aussi le type d'œuvres culturelles, la dynamique des utilités peut prendre des formes différentes. D'une façon générale, on peut distinguer trois schémas explicatifs emboîtés, avec la fonction d'utilité $U i[$.] représentant les goûts et pour laquelle $Q i$ représentera le bien culturel consommé par le consommateur $i$, Ki, le capital culturel constitué par l'individu $i$ au cours de ses consommations antérieures et $K$ le capital culturel social :

- soit l'utilité de chaque consommateur dépend d'une sorte de capital culturel qui dépend de ses consommations passées et fait de lui un amateur plus ou moins compétent $U i[Q i, K i]$. À l'écoute d'une œuvre radicalement nouvelle, chaque consommateur se trouve donc devant un choix d'investissement : vaut-il mieux consommer une œuvre d'un genre déjà connu ou est-il avantageux d'investir dans la constitution d'un nouveau capital ? L'utilité de court terme (Ui[Qi, 0]) indique mal ce que pourraient être l'utilité de long terme et les capacités d'apprentissage. Il s'agit donc d'un cas aggravé de bien d'expérience : il faut parier ex ante non seulement sur l'utilité qu'apportera le bien ex post mais encore sur son éventuelle utilité de long terme, après une acculturation que le consommateur ne saurait imaginer;

- soit l'utilité de chaque consommateur dépend d'une sorte de capital culturel social, l'appréciation d'une œuvre nouvelle demandant une sorte de travail collectif, d'appropriation sociale, généralement au sein d'une micro-culture plus ou moins structurée $(U i[Q i, K])$. Pour chaque consommateur, le pari est initialement encore plus risqué que dans le cas précédent puisque chacun ignore ce que vont faire les autres (problème de coordination) mais, une fois le mouvement lancé, les échanges entre consommateurs et la constitution d'une micro-culture facilitent l'acculturation de chacun ;

- soit, enfin, l'utilité de chaque consommateur dépend de ses capacités à contribuer, même marginalement, au capital culturel social en imitant ou en reprenant des œuvres nouvelles ( $U i[Q i, K i$, $K])$; dans ces conditions, l'ouverture des œuvres et la capacité de les modifier et de les transformer sont essentielles (selon des modalités de type Creative Commons par exemple); le bien-être dépend alors non seulement de l'ensemble des œuvres mais de la combinatoire qu'elles peuvent induire à partir de leurs composants élémentaires.

\section{LES CINQ SCÉNARIOS}

Les scénarios proposés respectent un schéma de présentation qui va de la vision du bien musical à la structure du marché, en passant par la nature des rapports entre le système "production/diffusion/ promotion » et les consommateurs et artistes.

\section{Logique de constitution}

Les scénarios d'évolution de la filière de la musique enregistrée tentent de dessiner pour les années à venir une double transition : celle du changement de support pour les contenus, le support numérique se substituant progressivement au support physique ; celle de la transformation des systèmes de traitement de la méta-information nécessaire au développement d'une demande et d'une offre adaptées, internet venant enrichir les médias de masse et transformer leur logique centralisée.

Ces scénarios sont obtenus à partir de la variable « extraction de la valeur» (soit par protection, soit par déplacement) croisée avec une variable traduisant l'évolution du système de prescription, c'està-dire du système de circulation de la méta-information (voir tableau 3). Aujourd'hui principalement Star system, celui-ci représente plus des trois quarts du marché en valeur et pourra évoluer vers un des trois autres états et l'on obtiendrait trois types de transition probablement un peu caricaturaux :

- du Star system au Pull structuré : dans cette optique, le modèle actuel des labels indépendants s'impose ;

- du Star system au Push : le marché de la musique enregistrée est théoriquement ouvert à tous mais, en réalité, dans le modèle Push plus encore qu'actuellement, très peu d'artistes bénéficient de la promotion médiatique nécessaire pour se faire connaître ;

- du Star system au Pull libre : les maisons de disque - tout au moins dans la forme que nous leur connaissons aujourd'hui - disparaissent progressivement; elles sont remplacées par un système totalement décentralisé de mise en relation des artistes et de leur public.

\section{Tableau 3 - Dynamique des modèles d'affaires dans la filière de la musique enregistrée à l'ère du numérique}

\begin{tabular}{|lc|}
\hline & Sélection $\quad$ Pas de sélection \\
Promotion centralisée & Pull structuré
\end{tabular}


À partir de ce croisement, on obtient cinq scénarios contrastés (voir tableau 4). Le sixième scénario possible n'a pas été retenu car il est peu vraisemblable que la protection des contenus musicaux soit compatible avec le Pull libre, qui suppose essentiellement que ces derniers sont ouverts au réemploi, appropriables et modifiables.

\section{Tableau 4-Les cinq scénarios d'évolution de la filière de la musique enregistrée}

\begin{tabular}{|lcc|}
\hline & Mode d'extraction de la valeur \\
& P.v & p.Z \\
Star system vers Push & Hit and run & Jingle \\
Star system vers Pull structuré & Happy few & NetLabel \\
Star system vers Pull libre & & Consumartist \\
\hline
\end{tabular}

Ces cinq scénarios correspondent à une implication croissante des consommateurs dans la production musicale. Le passage d'une extraction de valeur par protection à une extraction par déplacement libère les contenus et autorise une plus grande liberté d'utilisation par les usagers, en particulier l'interopérabilité, la copie, l'échange. D'autre part, le passage du Push au Pull structuré donne aux consommateurs un rôle de producteurs de métainformation, tandis que le passage au Pull libre leur laisse une responsabilité plus ou moins grande dans la production même du contenu.

\section{Description des scénarios}

Les cinq scénarios sont décrits selon un schéma identique, constitué de sept critères :

- la vision du bien musical. Il s'agit de la vision dans le sens indiqué précédemment - musique comme bien standard, comme bien social, comme bien culturel à capital individuel ou collectif, comme bien contributif - qui sera dominante dans le cadre du scénario considéré ;

- la diversité des ceuvres. On distinguera entre diversité offerte et diversité finalement consommée. Il peut en effet se faire que, dans certains scénarios, la diversité de l'offre dépasse les capacités d'information et d'acculturation qui seraient nécessaires aux consommateurs pour qu'ils puissent en bénéficier pleinement. Dans de tels cas, la diversité des contenus devient inutile tant que ne sont pas en place les capacités de gestion de la méta-information qui permettent de gérer cette diversité et d'en tirer pleinement parti ;

- la source de la valeur et le recueil financier. La valeur peut être assise sur le bien lui-même, sur la promotion qui en est faite, sur la méta-information qui lui est liée ou sur la collaboration du consommateur à l'élaboration du contenu. Le recueil de cette valeur peut se faire par vente de biens physiques, de fichiers rivaux ou de services. Il peut également provenir de revenus publicitaires, ou d'abonnements à des sites. Il y a de moins en moins de rapport entre la source de la valeur et les modes de recueil de la disposition à payer des consommateurs (ou de la disposition à payer collective);

- le type d'asservissement entre demande et offre. On peut considérer en effet plusieurs régimes selon l'équilibre qui s'établit entre l'orientation à court terme de l'offre par une demande supposée préexistante et l'orientation à long terme de la demande par une offre originale et innovante ;

- la nature des rapports entre le système de production/diffusion/promotion, les consommateurs et les artistes. Ces rapports peuvent être conflictuels ou coopératifs : on distinguera ainsi des situations défavorables ou favorables aux consommateurs; de même, symétriquement, des situations défavorables ou favorables aux artistes. Ainsi, des situations où des producteurs menacent de prison certains consommateurs soupçonnés de piratage seront qualifiées de défavorables aux consommateurs; de même des situations où des producteurs cherchent à se passer d'artistes originaux pour imposer une production standardisée seront qualifiées de défavorables aux artistes;

- les acteurs clés. Il s'agit des acteurs qui seront en position de freiner efficacement ou d'accélérer le mouvement, soit qu'ils bénéficient à court terme des évolutions, soit, inversement, qu'ils craignent une remise en cause trop profonde de leurs modèles d'affaires ;

- l'activité et la structure du marché. Il s'agit de déterminer qui pourrait finalement s'imposer, une fois le scénario établi. On distinguera l'activité en volume et l'activité en valeur.

\section{(1) Le scénario Hit and run}

Les acteurs tentent de maintenir, aussi longtemps qu'il est possible, les modèles d'affaires prénumériques. Ils privilégient le maintien du mode d'extraction de la valeur à partir de biens physiques (comme le CD) ou de fichiers rendus artificiellement rivaux. Pour ce faire, un appareil répressif est mis en place pour endiguer le piratage. Il s'appuie sur des protections techniques (DRM) et sur un renforcement des mesures juridiques avec notamment des sanctions beaucoup plus dissuasives pour les pirates. Dans ce scénario, le changement de support dominant, du $\mathrm{CD}$ vers le fichier numérique, se fait lentement.

Le modèle d'affaire emblématique du Hit and run est la vente de titres sur la plate-forme iTunes 
constituée majoritairement de fichiers protégés et non interopérables. Il en résulte une diversité de consommation peu différente du modèle physique et donc relativement limitée. Les maisons de disques cherchent à produire des hits et pratiquent des tarifications de monopoles.

- Vision du bien musical. Au scénario Hit and run correspond une vision de la musique comme bien économique standard, sur lequel pèsent des droits de propriété analogues aux droits sur les biens rivaux. Les fichiers numériques sont verrouillés ou liés à un support physique. Ils ne doivent être ni copiés ni échangés ; tout au plus, dans certains cas, l'utilisateur peut-il copier le fichier sur deux supports différents (micro-ordinateur et baladeur, par exemple). Les DRM rendent la consommation difficile et interfèrent avec le fonctionnement des terminaux (en particulier, des micro-ordinateurs), ce qui pose des problèmes de confidentialité et de sécurité informatique. Dans cette optique, la musique ne peut être un vecteur de convivialité entre consommateurs.

- Diversité des œuvres. Dans ce scénario, la diversité offerte est moyenne (celle qui régnait dans le monde de la distribution physique lorsque existaient des disquaires dits «indépendants » et des surfaces spécialisées). La diversité consommée est, elle aussi, moyenne et sensiblement inférieure à la diversité offerte.

- Source et recueil de la valeur. Les fichiers protégés sont vendus sur des plates-formes de distribution sur internet. La valeur de la musique réside dans les contenus protégés et le recueil de cette valeur se fait à partir de la vente de ces quasiobjets. Pour que ce modèle s'impose il faut à la fois que les DRM soient efficaces et que leur contournement soit interdit de façon rigoureuse.

- Asservissement entre demande et offre. Le scénario Hit and run prolonge la situation actuelle, sans tenir compte des nouvelles opportunités offertes par la numérisation. En conséquence, comme à l'époque des seuls disques et de leur diffusion onéreuse, l'offre cherche à suivre dans le court terme une demande déjà exprimée afin de minimiser les risques financiers du lancement d'une œuvre originale. La frilosité et le manque d'originalité de la production sont justifiés par la mise en avant de l'objectif de suivre les goûts des consommateurs.

- Rapports entre le système de production/diffusion/promotion, les consommateurs et les artistes. Le système de production/diffusion/promotion est ici à la fois défavorable aux consommateurs et aux artistes. Les producteurs, les éditeurs, les distributeurs menacent les consommateurs de lourdes peines, voire de prison, s'ils copient des fichiers ou s'ils les téléchargent sur des réseaux de pair à pair. Les consommateurs, de leur côté, éprouvent pour la filière de la musique enregistrée ressentiment et colère. Les avantages de la numérisation sont à portée de la main et il est clair pour tous qu'un système qui peine à s'adapter freine le progrès technique. Les artistes, dans leur grande majorité, mais pas ceux qui, déjà très connus, bénéficient du système, souhaiteraient court-circuiter cette organisation malthusienne ; ils craignent, toutefois, s'ils diffusent leur musique sous une forme libre (par exemple selon une procédure Creative Commons) de ne plus pouvoir tirer profit de leurs œuvres une fois le succès venu.

- Acteurs clés. Dans le cadre du scénario Hit and run, les acteurs chargés du verrouillage du contenu jouent un rôle primordial. Il s'agit principalement des majors, des producteurs de terminaux et de supports, des concepteurs de DRM, des producteurs de lecteurs logiciels (comme Windows Media Player, Winamp...), éventuellement des opérateurs de réseaux, lorsque ceux-ci veulent «monter dans les contenus » à partir de leur protection (en développant des techniques de cryptage, comme France Telecom avec sa filiale Viaccess). Le plus souvent, les terminaux comme les lecteurs logiciels ne sont pas interopérables (baladeur Zune) et donnent lieu à d'importants gaspillages, au moins en termes de bien-être social, dus au développement de réseaux de distribution incompatibles.

- Activité et structure du marché. Le scénario Hit and run conduit à une baisse du marché en valeur et en volume :

- la baisse du prix de la musique enregistrée pratiquée ces dernières années ne peut être enrayée; il est en effet illusoire d'espérer aligner à long terme le prix des fichiers protégés sur le prix des supports physiques ;

- le passage d'un mode d'achat dominant par album à un mode par titre réduit vraisemblablement les recettes par artiste sans réduire les coûts dans la même proportion, dans l'hypothèse vraisemblable où l'élasticité-prix de la demande est telle que l'effet de la vente par paquet (bundling) n'est pas dominant ;

- un renforcement des DRM (de copie et d'usure) joint à une répression sévère du piratage produira des effets contre-productifs importants.

La baisse d'activité du marché de la musique enregistrée se répercute sur l'ensemble de la filière. Sans doute plus fortement sur les studios et les distributeurs que sur les éditeurs, dont la moitié seule- 
ment des revenus provient des ventes de musique enregistrée, et sur les entrepreneurs de spectacle.

Deux facteurs semblent devoir favoriser un renforcement de la concentration industrielle du secteur en fragilisant les labels indépendants :

- la disparition progressive des lieux physiques d'achat et d'information sur les nouveautés musicales, en particulier les disquaires indépendants ; - l'inefficacité de la promotion en ligne incapable de prendre à court terme le relais des garants physiques de la diversité.

\section{Le scénario Jingle}

Dans ce scénario, les acteurs ne cherchent pas à collecter la valeur créée par la musique enregistrée à partir de la vente de contenus protégés. Ils considèrent qu'il n'est pas souhaitable de chercher à endiguer le piratage et que les effets négatifs des DRM sur le développement du marché dominent leur effet protecteur. La musique enregistrée est alors distribuée gratuitement et les revenus de la filière sont captés sur d'autres marchés dont la croissance serait dopée par la baisse des prix des contenus.

- Vision du bien musical. Au scénario Jingle correspond une vision de la musique comme bien social. La musique est considérée comme sans valeur intrinsèque. Elle accompagne d'autres biens, dont elle sert la promotion. Elle pointe vers divers produits ou services dont elle rend la consommation plus probable. Elle peut enfin être consommée pour des raisons ostentatoires ou comme signal de l'appartenance à un groupe ou à une sous-culture. Ce qui est caractéristique du scénario Jingle, c'est que le succès d'une œuvre et le profit qu'elle génère ne dépendent pas essentiellement de l'œuvre elle-même et de sa qualité mais seulement de la promotion dont elle bénéficie et du rôle économique et social qu'elle joue.

- Diversité des œuvres. Dans le cadre du scénario Jingle, la diversité offerte à un moment donné est très faible, même si l'offre est susceptible de se renouveler rapidement. Au reste, il convient de distinguer le renouvellement des titres et celui des genres. Les œuvres dont les médias de masse font la promotion se renouvellent rapidement par effet de mode mais des genres vraiment nouveaux émergent difficilement.

- Source et recueil de la valeur. Dans le cadre d'une économie de l'attention ${ }^{10}$, la musique tire l'es-

10. Une économie de l'attention est une économie où les biens et services acquièrent de la valeur parce qu'on attire vers eux l'attention du public. Ils restituent en partie cette valeur en attirant, à leur tour, l'attention du public vers des biens et services dérivés. sentiel de sa valeur de la promotion par les médias de masse. Cette valeur peut être recueillie soit dans les produits dérivés, comme par exemple la sonnerie d'un téléphone portable, soit à partir de publicités, souvent agressives, par exemple l'écoute de spots publicitaires pendant un morceau de musique (le projet Spiral Frog donne une première idée de ce type de publicité en prévoyant l'écoute obligatoire d'une publicité avant chaque morceau).

- Asservissement entre demande et offre. Dans le cadre du scénario Jingle l'offre n'a même plus à chercher à suivre la demande à court terme. L'efficacité de la promotion par les médias de masse est telle que n'importe quelle œuvre, n'importe quel artiste, même un amateur pris au hasard, aura un succès qui ne sera fonction que de l'intensité de la promotion. Il n'y a pas d'asservissement de l'offre à la demande, tout simplement parce que, dans ce scénario, il n'y a ni goûts ni demande qui ne soient produits, directement ou indirectement, par les médias de masse.

- Rapports entre le système de production/diffusion/promotion, les consommateurs et les artistes. Dans ce scénario le système de production/diffusion/promotion est plutôt favorable aux consommateurs puisque les œuvres musicales ne sont pas mises en valeur à partir de fichiers protégés par des $D R M$ gênantes et la criminalisation de la consommation. En revanche, les artistes comme les labels indépendants considèrent que les médias formatent les goûts des consommateurs et promeuvent une musique sans qualité, une sorte de musique d'ascenseur, peut-être même élaborée bientôt par des logiciels. Il s'agit donc d'un scénario défavorable aux artistes.

- Acteurs clés. Pour le scénario Jingle, les acteurs clés sont d'une part les médias de masse, qui pourront d'ailleurs créer leurs propres labels (comme Music One pour TF1) ou leurs propres sites de commercialisation (M6music pour M6 ou Urge MTV pour MTV); d'autre part, les majors et les plates-formes de distribution en ligne financées par la publicité. De tels sites produisent, selon le même modèle que les médias, tout à la fois des biens culturels formatés et les audiences qui leur correspondent. Ils diffusent en continu (streaming) de la musique coupée par des spots de publicité.

- Activité et structure du marché. Dans un scénario où la consommation de musique enregistrée est dictée par les médias de masse, les plus gros acteurs sont avantagés parce qu'ils sont les seuls à avoir accès à une promotion efficace. Ils sont également plus à même de négocier des contrats 
satisfaisants avec les partenaires des marchés dérivés ${ }^{11}$

Le marché ira donc en se concentrant et les labels indépendants connaîtront des difficultés croissantes à conclure des contrats équitables. Les modifications pourraient être plus profondes encore si certains acteurs des marchés de l'accès choisissaient de s'intégrer verticalement dans la production musicale, comme ils l'ont déjà fait dans la distribution. De ce point de vue, une contribution des opérateurs de l'accès et des industriels au financement de la création musicale (taxe sur les fournisseurs d'accès à internet, extension de la copie privée, etc.) aurait un effet stabilisateur; une telle contribution pourrait même être bénéfique si elle était dotée d'effets redistributifs vers les nouveaux artistes et les plus petits acteurs. L'activité est cependant difficile à prévoir en volume comme en valeur puisqu'elle dépend de la consommation sur d'autres marchés. Soulignons d'ailleurs que les sommes en jeu ne seraient vraisemblablement pas négligeables ${ }^{12}$.

\section{Le scénario Happy few}

L'efficacité de la promotion décentralisée en ligne offre de nouvelles opportunités aux acteurs qui n'ont plus à s'appuyer nécessairement sur les médias centralisés pour promouvoir les œuvres auprès du public. Le modèle de référence est le Pull structuré.

Si la vente de fichiers protégés et non interopérables reste possible dans le cadre de ce scénario, la protection des contenus prend des formes plus variées et plus subtiles que dans le scénario précédent : adressage, segmentation, $D R M$ de péremption plutôt que de copie, etc.

La diversité d'offre et de consommation est plus élevée qu'aujourd'hui. Il pourrait en résulter une distribution numérique moins concentrée avec des acteurs susceptibles de se positionner sur des niches de marché avec, comme avantage comparatif par rapport à des acteurs généralistes comme la plateforme iTunes, d'enrichir la vente d'un fichier numérique par la fourniture de recommandations statistiques ou communautaires.

\footnotetext{
11. Il n'est pas anodin que seul Universal Music, et non l'ensemble des acteurs de la production musicale, soit parvenu à conclure un accord avec Microsoft conduisant à un reversement d'une fraction - certes modeste - des recettes réalisées par le baladeur Zune.

12. À titre d'exemple, une taxe mensuelle de $2 €$ collectée sur chaque abonnement à l'internet haut débit (qui rapporterait environ 260 millions d'euros) pourrait compenser plus des trois quarts de la baisse du chiffre éditeurs de la production musicale enregistrée entre 2002 et 2005 (340 millions d'euros selon le SNEP).
}

- Vision du bien musical. Au scénario Happy few correspond une vision de la musique comme bien culturel à capital individuel. Les amateurs, chacun individuellement, consomment les musiques qu'ils apprécient mais aussi s'initient à des œuvres innovantes et s'acculturent progressivement à des genres nouveaux. Les contenus sont payants et les essais d'œuvres nouvelles sont donc onéreux. Les réseaux de pair à pair libres sont pris en main par des maisons de disques ou des distributeurs en ligne et servent à la fourniture payante de fichiers. Le comportement de découverte de musiques nouvelles est réservé à une minorité : ceux qui ont les moyens de parier sur l'utilité que leur apporteront, éventuellement, de telles œuvres.

- Diversité des œuvres. La diversité des œuvres offertes peut être grande, en particulier si les labels indépendants et les distributeurs en ligne jouent leur rôle. Toutefois, la diversité effectivement consommée est sans doute très inférieure à ce qu'elle pourrait être en raison des prix encore élevés des fichiers numériques.

- Source et recueil de la valeur. Comme dans le scénario Hit and run, la valeur de la musique réside dans les contenus. Mais dans ce scénario, le recueil de la valeur est plus inventif : s'il peut encore avoir lieu à partir de la vente de fichiers protégés, le plus souvent, les producteurs et les distributeurs recourent ainsi à diverses stratégies commerciales :

- la vente par paquets (bundle) larges qui revient à la fourniture gratuite d'œuvres non encore appréciées ;

- l'adressage personnel à partir de système statistique de recommandation renseigné par la consommation passée (chaînes de diffusion qui « apprennent» les goûts et présentent des œuvres nouvelles susceptibles de plaire, comme Musicmatch, Last.fm, Emosound, Pandora) ;

- l'adressage temporel (DRM de péremption), par exemple par la fourniture gratuite, en quelque sorte «à l'essai », d'œuvres nouvelles.

- Asservissement entre demande et offre. Au moins pour une clientèle aisée, le scénario Happy few permet à des musiques nouvelles de trouver leur public. Ce scénario tente d'asservir la demande à l'offre et non l'inverse, sortant ainsi du raisonnement caricatural «il faut donner aux consommateurs ce qu'ils aiment; or ils aiment de la musique médiocre, donc c'est ce qu'il faut leur donner; de plus il s'agit là d'une musique peu onéreuse à produire, c'est donc tout bénéfice ». Le risque existe, toutefois, que ne se constitue une sorte de fracture dans le public entre ceux qui pourront bénéficier 
d'un modèle « payant » de qualité et ceux qui resteront dans le modèle «gratuit» des médias (décrit précédemment sous le nom Jingle).

- Rapports entre le système de production/diffusion/promotion, les consommateurs et les artistes. Le scénario Happy few est en quelque sorte intermédiaire. Il n'est pas défavorable aux consommateurs comme le scénario Hit and run. Il n'est pas non plus défavorable aux artistes comme le scénario Jingle. Mais il ne peut pas être qualifié de favorable aux consommateurs ou aux artistes comme les scénarios qui vont suivre. C'est un scénario de compromis qui ne tourne pas le dos au progrès technique mais qui n'en tire pas non plus tous les avantages afin de ménager les modèles d'affaires actuels.

- Acteurs clés. Dans le cadre du scénario Happy few, les labels indépendants, éventuellement regroupés, jouent un rôle clé. Plus généralement, les acteurs capables d'assurer une grande diversité de la production et de la distribution ainsi qu'une tarification inventive, sont essentiels et structurent la filière: distributeurs spécialisés (FNAC Music, VirginMega), plates-formes de distribution en ligne de fichiers plus ou moins protégés, selon un modèle dont les plates-formes e-compil ou Buy Music donnent des exemples.

- Activité et structure du marché. L'activité devrait croître, certainement en volume, peut-être en valeur, en raison d'une bonne adaptation de l'offre et de la demande. Toutefois, cet effet pourrait être contrebalancé par l'effet dissuasif de prix élevés au regard d'éventuelles contraintes d'utilisation imposées par les DRM et la non-interopérabilité. S'agissant de la structure de marché, le modèle des labels indépendants, privilégiant la recherche de talents, devient le modèle de référence tandis que recule celui du Star system. Les majors déclinent tandis que des labels indépendants se regroupent pour réduire leurs frais généraux. Au niveau de la distribution, à côté des nouveaux venus mentionnés plus haut, la domination de quelques acteurs non issus de la filière (Apple, Microsoft, opérateurs de téléphonie) s'affirme au détriment des distributeurs historiques. De même, les studios, les éditeurs et les producteurs de spectacle connaissent peu de changements.

\section{Le scénario NetLabel}

Devant la diversité croissante de la production, la valeur ne réside plus dans les contenus musicaux mais dans les procédés techniques et les processus sociaux qui permettent la circulation efficace de la méta-information.
L'efficacité de la promotion décentralisée sur internet met la recherche de talents au centre de l'activité de la production musicale. La promotion centralisée par les médias est remplacée par une promotion assurée par des experts moins autoproclamés que reconnus par les internautes, ce qui permet une meilleure adéquation entre la production musicale et la demande.

Dans un tel scénario, le financement publicitaire perd de sa pertinence puisqu'il y a un plus grand nombre d'artistes et chacun dispose d'une audience plus réduite que dans le scénario Jingle. Au-delà de la vente de fichiers sur internet, chaque artiste peut être financé par le partage des recettes de concerts et par du marchandisage, ce qui serait d'autant plus pertinent que les investissements initiaux se verraient amputés des frais de promotion et de distribution physique. Un financement global de la filière par une contribution en provenance des marchés d'accès est aussi envisageable.

- Vision du bien musical. Au scénario NetLabel correspond une vision de la musique comme bien culturel à capital social. Les consommateurs échangent souvent des opinions sur les œuvres qu'ils consomment. Ils se connaissent parce qu'ils appartiennent à une même communauté réelle ou virtuelle et ils se font suffisamment confiance pour suivre les conseils de leur communauté concernant les œuvres nouvelles.

- Diversité des œuvres. La diversité offerte est très grande. Elle se prolonge ou non par une grande diversité consommée selon l'efficacité des mécanismes d'acculturation communautaire qui se mettent en place.

- Source et recueil de la valeur. Le scénario NetLabel se caractérise par le fait que la valeur de la musique enregistrée réside essentiellement dans la méta-information nécessaire à sa consommation. Le recueil de cette valeur pose problème. D'une part, puisqu'il s'agit de méta-information, il s'agit d'un bien adressé (utile à un seul consommateur) qui pourrait théoriquement être mis en valeur directement par vente ou abonnement; d'autre part, l'habitude n'est pas de vendre la méta-information, en particulier parce que sa valeur dépend de l'objectivité avec laquelle elle est élaborée. Mais des contre-exemples existent : Google, par exemple, a trouvé un modèle efficace de séparation entre la méta-information et la publicité.

- Asservissement entre demande et offre. Au modèle NetLabel correspond une orientation de la demande par une offre novatrice. On notera cependant que le caractère communautaire de l'appropriation des œuvres peut conduire à une 
remontée d'information de la demande vers les labels et les artistes. Il y a asservissement à court terme de la demande à l'offre mais, à long terme, l'innovation musicale pourrait, dans une certaine mesure, être asservie à la demande.

- Rapports entre le système de production/diffusion/promotion, les consommateurs et les artistes. D'une façon générale, le système de production/diffusion/promotion est favorable aussi bien aux consommateurs qu'aux artistes. On notera toutefois que le succès du scénario dépend plutôt des rapports entre diffuseurs et consommateurs que des rapports entre producteurs et artistes. En conséquence, si un tel scénario s'impose, le système production/diffusion/promotion sera sans doute plus favorable aux consommateurs qu'aux artistes.

- Acteurs clés. Pour le scénario NetLabel, les industriels et les vendeurs d'équipements, en particulier les équipements de home studio, ainsi que les plates-formes d'interaction sur internet jouent un rôle clé. Ces dernières ont plus spécifiquement la responsabilité de créer un «écosystème » qui permette une invention et une acculturation congruentes. Divers modèles sont envisageables pour rapprocher artistes, labels indépendants, amateurs et consommateurs :

- fourniture de fichiers non protégés (comme Starzik, MusicMe, Bleep ou eMusic);

- fourniture de méta-informations sous la forme de bases de données financées par la publicité (par exemple, des bases de données comme Allmusic, Discogs, ou l'agrégation de blogs comme Hypemachine) ;

- organisation de réseaux sociaux de prescription (comme, par exemple, Amie Street, BnFlower ou même $M y S_{\text {Sace }}{ }^{13}$ ); certains sites cherchent aussi à recueillir la valeur des réseaux de prescription, selon un modèle de financement des prescripteurs (Weed) ;

- fourniture de méta-informations sous forme de navigation (par essais-erreur) dans l'espace des goûts à partir de systèmes statistiques de recommandation (comme, par exemple, Emosound, Last.fm, Pandora, Musicmatch).

- Activité et structure du marché. Dans ce scénario, l'activité en volume croît rapidement ; en valeur, l'incertitude demeure sur l'inventivité et l'efficacité des modèles qui reposeront sur la mise en valeur de la méta-information.

13. L'accord récent entre un regroupement de maisons de disques indépendantes, Merlin et MySpace, au travers du gestionnaire de licences MyStore, fournit une illustration des relations qui se mettent en place dans le cadre de ce scénario.
Un grand nombre de petits labels, plus ou moins indépendants, sont susceptibles de coexister. La promotion décentralisée permet de faire émerger des segments de demande homogène. Les barrières à l'entrée se trouvent singulièrement réduites par la baisse des frais de promotion et de distribution physique. La recherche de talents devient cruciale, du fait de l'élargissement de l'offre.

À une meilleure adéquation de l'offre et des goûts des consommateurs devrait correspondre une plus forte disposition à payer qui se traduirait par des dépenses plus élevées sur les marchés dérivés et, en retour, par un financement plus important de la création musicale.

\section{Le scénario Consumartist}

Dans le scénario Consumartist, les différences entre professionnels, producteurs occasionnels et amateurs s'estompent fortement. Une part de la valeur reste non marchande. Plus encore que dans les autres scénarios, les gains de bien-être ne se résument pas aux profits et aux surplus des acteurs ; il s'agit d'une transformation profonde de la façon de produire et de consommer la musique.

Inversement, il faut considérer que ce scénario demande, en raison des importantes évolutions économiques, sociales et juridiques qu'il nécessite, une période de transition longue et inconfortable pour certains acteurs de la filière. De plus, la coexistence d'un modèle ouvert de création collective avec le modèle dominant actuellement (celui des médias de masse) peut susciter des espoirs infondés. Une plate-forme comme MySpace, qui appartient depuis 2005 à Newscorp, groupe de Rupert Murdoch, peut donner l'illusion d'un accès facile pour tous à une promotion efficace alors qu'il s'agit plutôt d'une extension des médias de masse. Les labels les plus importants utilisent aujourd'hui MySpace et les échanges sur les réseaux de pair à pair pour tester les différents titres d'un album et évaluer leur adéquation aux goûts du public.

- Vision du bien musical. Au scénario Consumartist correspond une vision de la musique comme bien culturel contributif. L'utilité des consommateurs dépend non seulement du capital social auquel ils ont accès, c'est-à-dire de leur acculturation obtenue au sein de communautés d'amateurs, mais aussi de leur éventuelle contribution à ce capital. L'ouverture ${ }^{14}$ des œuvres permet à chacun de les citer, de les modifier et de les réutili-

14. On entend ici par « ouverture », non seulement l'absence de DRM mais aussi la possibilité de réemployer une œuvre dans une nouvelle création originale (il s'agit de la notion d'ouverture au sens de « code ouvert » des logiciels libres). 
ser partiellement. Tous les consommateurs ne sont pas des amateurs éclairés et tous les amateurs éclairés ne sont pas des artistes, bien entendu, mais tous sont, au moins potentiellement, des contributeurs.

- Diversité des œuvres. La diversité offerte n'est peut-être pas sensiblement supérieure dans ce scénario à ce qu'elle est dans le scénario NetLabel mais la diversité consommée l'est certainement. Elle repose sur une sorte de combinatoire des œuvres disponibles, chaque consommateur recomposant comme il l'entend les formes qui lui plaisent et les mettant à la disposition de tous.

- Source et recueil de la valeur. Dans un tel scénario, la source de la valeur est double : d'une part, certaines œuvres susceptibles d'être réutilisées (des sortes de «formants », analogues aux briques blanches dans le domaine des services); d'autre part, la capacité des amateurs à les utiliser et à les recomposer. Le recueil peut donc se faire à partir, à la fois, des œuvres réutilisées (par des licences du type Creative Commons) et des services de formation aux activités de composition.

- Asservissement entre demande et offre. Au modèle Consumartist, correspond une coévolution de la demande et de l'offre dans la mesure où les notions de consommation et de production ont tendance à se confondre. On assiste alors, dans le domaine de la création musicale, à un développement des algorithmes de consommation et à une transformation des algorithmes de production assez semblables à l'évolution générale de désintermédiation qui existe dans d'autres secteurs économiques. De même que, dans le domaine du tourisme, les consommateurs peuvent composer leurs voyages en assemblant des services bruts (voyages, hôtels...) et « court-circuiter» les agences de voyages, de même, dans le scénario Consumartist les consommateurs composeront des œuvres en réutilisant des productions brutes. Un site comme YouTube ou, dans un autre domaine, Flickr, préfigure une telle économie du bricolage artistique.

- Rapports entre le système de production/diffusion/promotion, les consommateurs et les artistes. Comme dans le scénario précédent, le système de production/diffusion/promotion est aussi bien favorable aux consommateurs qu'aux artistes. On notera, toutefois, que le succès du scénario dépend de la façon dont les artistes confirmés considéreront la « concurrence» des amateurs. Si, de plus, le législateur, dans le but de défendre les artistes professionnels qui risqueraient d'être exploités, requalifie la production et les spectacles des amateurs en production professionnelle, le scénario Consumartist sera considéré, au moins par certains, comme défavorable aux artistes.

- Acteurs clés. Pour le scénario Consumartist, les acteurs principaux sont, outre les artistes euxmêmes, les plates-formes de commercialisation de services et d'éléments réutilisables, ainsi que les vendeurs d'équipements de home studio. Les opérateurs de réseaux pourraient jouer un rôle décisif, s'ils choisissent de «monter dans le contenu » à partir de l'organisation de réseaux de pair à pair efficaces et enrichis de plates-formes de gestion de la méta-information (communautés d'expérience et de pratique). Enfin, naturellement, les plates-formes de commercialisation de fichiers ouverts sous licences Creative Commons, comme aujourd'hui Jamendo ${ }^{15}$ ou Magnatune, ainsi que les sites qui tentent de créer des relations directes entre artistes et amateurs (Artistshare, Sell a $B a n d)$ deviennent des acteurs importants pour la recomposition de la filière.

- Activité et structure du marché. L'activité en volume croît fortement. Les circuits de mise en valeur restent à inventer. Ils sont ici plus complexes que ceux nécessaires à la valorisation de la méta-information. La notion d'auteurs perd progressivement son sens de même que la notion de propriété intellectuelle. Une telle évolution qui peut être, par certains côtés, enthousiasmante, risque toutefois d'aboutir à des conflits d'intérêt insolubles et des coûts de transaction prohibitifs si la définition des droits de propriété intellectuelle n'évolue pas substantiellement.

L'émiettement total de la production et la généralisation d'un modèle artisanal risquent paradoxalement d'être néfastes pour les stades professionnels de la filière : majors et gros labels, studios, distributeurs et éditeurs.

Les cinq scénarios théoriques peuvent trouver des éléments de consolidation à l'observation de certains nouveaux modèles d'affaires émergents dans la filière de la musique enregistrée. Sans prétendre à l'exhaustivité, le tableau mis en annexe (p. 16) présente quelques exemples de ces nouveaux modèles et les positionne par rapport aux cinq scénarios. On trouvera par ailleurs dans Fing ${ }^{16}$ une présentation très complète de ces nouveaux modèles économiques de la filière de la musique enregistrée à l'ère du numérique.

15. Les négociations actuelles entre Jamendo et la SACEM donnent un exemple des difficultés que rencontrera la musique numérique pour s'imposer comme bien culturel circulant.

16. Fing, Débat public: musique et numérique, créer de la valeur par l'innovation. Fédération internet nouvelle génération, 2007. http://musique.fing.org/files/Etat_Etude_Musique_FING.pdf. 


\section{CONCLUSION}

La crise que vit aujourd'hui le secteur de la musique enregistrée est paradoxale. En effet, face à une baisse continue des ventes de CD musicaux depuis le début des années 2000, jamais la musique n'a été autant écoutée et aimée ; jamais la production n'a été à ce point diversifiée ; jamais la distribution, aussi universelle. La musique est omniprésente. Elle est goûtée pour elle-même : les concerts attirent de plus en plus de spectateurs. La musique devient aussi signe social, depuis les sonneries des téléphones portables jusqu'aux ambiances musicales sur les sites internet.

Non seulement la musique est consommée en plus grande quantité mais elle est aussi mieux choisie et mieux adaptée aux goûts de chacun. La métainformation s'enrichit et gagne en efficacité : le bouche-à-oreille autrefois limité à un cercle intime s'étend aux communautés en ligne, c'est-à-dire à des réseaux immenses comme ceux qui se développent sur MySpace. Les modèles statistiques de prescription gagnent en finesse; certains sites offrent à chaque consommateur la possibilité de se déplacer dans un espace d'œuvres qu'il ne connaît pas encore mais qui correspondront à ses goûts. Bien mieux, on peut lui présenter un cheminement d'apprentissage qui lui permettra de s'acculturer à de nouveaux genres musicaux à partir de systèmes personnels d'écoute (par exemple, des logiciels sur des baladeurs) ou de sites adaptatifs de diffusion en streaming.

Lorsque les CD sont remplacés par des fichiers numériques, les coûts de production, dans une certaine mesure, et surtout les coûts de distribution, se réduisent fortement. De tels gains de productivité permettent une consommation plus diversifiée ainsi que le développement de pratiques intermédiaires entre la consommation et la production : les amateurs peuvent accéder à une qualité professionnelle pour les coûts limités d'un home studio. Les œuvres se désacralisent. Elles se réemploient, se composent avec d'autres, se réorganisent en œuvres nouvelles. Compilation, remix, sampling sortent des limites étroites du droit d'auteur comme les échanges sur les réseaux de pair à pair interrogent l'exception pour copie privée.

Pourtant, les processus de production, de distribution, de promotion tardent à tirer parti de ce que le progrès technique, internet et la numérisation permettent. Les consommateurs évoluent plus rapidement : ils contournent les interdictions et inventent de nouveaux modèles. Il y a donc urgence à ce que les modèles d'affaires, les habitudes de gestion, les contraintes juridiques évoluent pour permettre de mettre à profit les opportunités offertes par les techniques numériques plutôt que de n'en subir que les menaces.

Ces évolutions se traduiront vraisemblablement par une véritable révolution dans deux domaines : le mode de création de valeur dans la filière musicale et son mode de financement.

Le centre de gravité de la création de valeur se déplace des contenus vers la méta-information nécessaire à leur consommation ${ }^{17}$. Lorsque toute la musique enregistrée sera fournie avec l'achat d'un baladeur de même que les micro-ordinateurs sont équipés d'un système d'exploitation, la valeur des contenus viendra clairement des moyens logiciels et sociaux de naviguer parmi ces fichiers et de trouver ce qui peut plaire et surprendre. Dans un monde d'abondance des informations, la prescription et la méta-information créent la valeur des contenus. De ce point de vue, le mode de prescription des médias de masse et celui de la promotion décentralisée en ligne par l'intermédiaire du bouche-à-oreille électronique sont susceptibles de donner des résultats bien différents. Toutefois, si les divers acteurs de la filière musicale critiquent sévèrement le rôle prescripteur des médias de masse, qui réduisent la diversité et la qualité de la production, s'ils regrettent la disparition progressive des disquaires et craignent celle des grandes surfaces spécialisées, ils n'ont pas encore une vision précise du système de prescription qui se met en place sur internet à partir des plates-formes d'interaction du «web 2.0 ».

Lorsque la valeur de la musique dépendait des contenus et que la technique ne permettait pas leur copie et leur diffusion à des coûts très faibles, le recueil de cette valeur sur le marché pouvait financer la création musicale. Si la valeur de la musique repose désormais, au moins en partie, sur la métainformation, de nouveaux problèmes se posent : le mode de recueil de cette valeur pour financer les plates-formes, éventuellement les contenus; la mesure des audiences à partir de la consommation de méta-information; et, finalement, la recherche d'un financement de la création par des transferts de recettes depuis les activités qui dépendent plus ou moins directement de la musique : produits dérivés, industries des terminaux et des consommables, industries des réseaux.

17. P. ChANTEPIE et A. Le DiBERDER, Révolution numérique et industries culturelles, op. cit. 


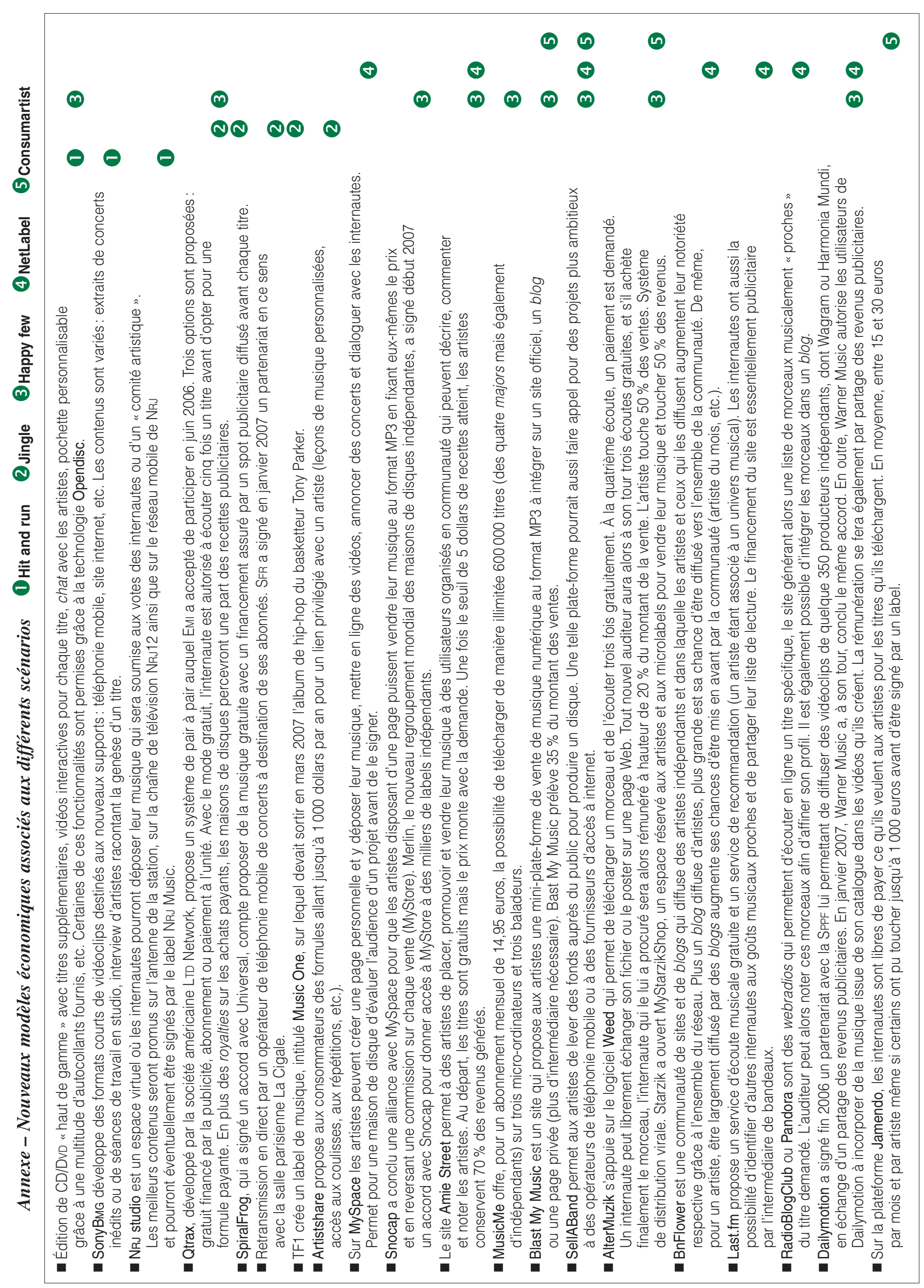

